\title{
Patients' feedback on the Changi General Hospital tinnitus counselling protocol
}

\author{
Joyce Jeanne Bee Hong $\underline{\operatorname{Lim}}^{1}$, GradDip, Peter Ku Sun $\underline{\mathrm{Lu}}^{1}$, MBBS, FRCS, David Soo Quee $\underline{K o h}^{1,2,3}$, MBBS, PhD
}

INTRODUCTION Many tinnitus service providers advocate counselling as an initial and important approach for the management of tinnitus. Our tinnitus counselling clinic (TCC) developed a counselling protocol to deliver information and educate patients on self-help strategies. We aimed to obtain feedback on the components of the initial session and the self-help choices made by patients who returned for follow-up.

METHODS All patients who were attending the TCC completed a Tinnitus Handicap Inventory (THI) questionnaire during their first and follow-up sessions. At follow-up, patients were asked to rate how helpful each counselling component was and their choice of self-help strategy for tinnitus.

RESULTS 203 patients attended the follow-up session, representing $60.1 \%$ of patients initially counselled. THI scores improved, with $45.8 \%$ more patients categorised as having no handicap at follow-up compared to the initial $26.1 \%$. Two most helpful counselling components (rated on a 5-point scale) were explanation of self-help strategies (mean score $4.40 \pm 0.68$ ) and illustration of healthy and damaged hair cells (mean score $4.30 \pm 0.74$ ). The most popular choice for self-help was using a sound strategy plus change in thinking $(41.9 \%, n=85)$. A few $(32.5 \%, n=66)$ patients were able to change their perception of tinnitus without any sound enrichment, while $17(8.4 \%)$ patients relied solely on sound strategy. Most (97.5\%) patients found the initial counselling session helpful. However, 92 (45.3\%) patients were still seeking a cure for their tinnitus.

CONCLUSION Most patients found the counselling helpful at follow-up. Counselling offered them the opportunity to actively manage their tinnitus.

Keywords: counselling, tinnitus, Tinnitus Handicap Inventory

\section{INTRODUCTION}

Tinnitus is a troubling symptom, often without a definite aetiology or known pathophysiology. As tinnitus is not always associated with hearing loss, when all audiological assessment and investigation results are within normal ranges, the attending doctor often advises patients to 'just live with it'.(1) Patients' quality of life can be severely affected, both emotionally and mentally; they may suffer from sleepless nights, loss of concentration, stress and paranoia.

Over the past decades, tinnitus management has included tinnitus retraining therapy, ${ }^{(2)}$ cognitive behavioural treatment, ${ }^{(3)}$ acupuncture, ${ }^{(4)}$ medications, ${ }^{(5)}$ tinnitus devices (e.g. Neuromonics) ${ }^{\left({ }^{(6)}\right.}$ ear implants, ${ }^{(7)}$ hearing aids, ${ }^{(8)}$ repetitive transcranial magnetic stimulation ${ }^{(9)}$ and auditory perceptual training. ${ }^{(10)}$ Many of these diverse interventions may provide some temporary relief for most tinnitus patients but are not necessarily effective for its total resolution. Some studies have found counselling to be a useful approach for helping patients to cope with tinnitus-induced anxiety and depression as well as expectations of a cure. ${ }^{(11,12)}$

To help patients with tinnitus better cope with their condition, a Tinnitus Counselling Clinic (TCC) was established at Changi General Hospital (CGH), Singapore, in 2008. The initial CGH tinnitus counselling protocol delivered information to the patient about the ear, the process of hearing, and the causes and mechanisms of tinnitus. Concomitantly, the tinnitus counsellor attempted to allay the patient's fears and concerns, and impart some self-help strategies. This enabled the patient to be well informed about tinnitus and empowered them to cope and accept the condition more readily.

The objective of this study was to: (a) obtain and review routinely collected patient feedback on the $\mathrm{CGH}$ tinnitus counselling protocol; (b) study which component(s) of the protocol the patients considered helpful; and (c) determine the choices for self-help management after initial counselling of patients who returned for follow-up.

\section{METHODS}

An operational review was conducted of the routinely collected clinical records of all patients with tinnitus who underwent counselling at the TCC at CGH from January 2010 to January 2013 and who attended subsequent follow-up sessions. There was no exclusion criteria. All patients with tinnitus who attended the tinnitus counselling first underwent a routine ear, nose and throat (ENT) consultation. Clinical history was taken, and visual inspection of the ears and otoscopic examination was performed by ENT doctors from the Department of Otolaryngology. Compulsory pure-tone audiogram and tympanogram were performed by audiologists from the same department. All findings were assessed and explained to the patients by the ENT doctors

${ }^{1}$ Department of Otolaryngology, Changi General Hospital, ${ }^{2}$ Saw Swee Hock School of Public Health, National University Health System, National University of Singapore, Singapore, ${ }^{3}$ PAPRSB Institute of Health Sciences, University Brunei Darussalam, Brunei

Correspondence: Ms Lim Bee Hong Joyce Jeanne, Senior Tinnitus Counsellor, Department of Otolaryngology, Changi General Hospital, 2 Simei Street 3 , Singapore 529889. joyce_lim@cgh.com.sg 
prior to referral to the initial counselling session. The counselling session was conducted by the main author, who was the sole tinnitus counsellor and Tinnitus Handicap Inventory $(\mathrm{THI})^{(13)}$ administrator in the hospital. The self-reported THI questionnaire is a 25-item questionnaire that has three responses to each question. A 'yes' response scores 4 points, 'sometimes' 2 points, and 'no' 0 points. The total score, which has a range of $0-100$ points, is classified into four THI handicap categories: none (0-16 points); mild (18-36 points); moderate (38-56 points); and severe ( $\geq 58$ points).

The initial counselling session lasted 90-120 minutes and consisted of six components: (1) administration of the THI and tinnitus history taking; (2) introduction about the ear and hearing, causes of tinnitus and related theories; (3) interpretation of patient's audiogram; (4) creating a better understanding of healthy and damaged cochlear hair cells by showing pictures using a toothbrush analogy (Fig. 1); (5) explaining sound and non-sound self-help strategies; and (6) providing written recommendations tailored to the individual patient. The sound strategies used were: (a) sound enrichment environment (i.e. opening windows, switching on the fan or air-conditioning to enrich the environmental soundscape); and (b) listening to radio programmes to improve hearing skills, engage brain activities (e.g. attention, understanding, memory, emotions and reactions) and promote natural habituation to the tinnitus. Nonsound strategies included: (a) detecting trigger factors (e.g. lack of sleep, stress, feeling unwell, negative emotions, stressors and more hearing loss as trigger factors) so that the patient could learn to manage the trigger factors and not the tinnitus; (b) distraction and relaxation (e.g. physical exercises, watching television and cycling); and (c) changes in thinking, as tinnitus is not harmful and will not cause deafness. While we may be unable to remove tinnitus, the patient can stem his or her reactions to it and learn to treat tinnitus as part of the background noise and ignore it; maintaining a happy mind would also help.

All patients were given a three-month follow-up appointment. The THI questionnaire was again completed during the follow-up session. At the same time, a feedback questionnaire was administered to rate how helpful each counselling component was to the patients (on a 5-point scale ranging from least to most helpful) and determine their choice of sound or non-sound strategies for tinnitus management. Patients were further asked if they found counselling helpful and if they were still seeking a cure for their tinnitus.

$\mathrm{THI}$ and feedback questionnaires were evaluated by the lead author. Nominal, ordinal and numeric data was entered into a database for statistical analysis. Qualitative comments were selected by the lead author for inclusion in the study based on their relevance and impact.

SPSS version 16.0 (SPSS Inc, Chicago, IL, USA) was used to analyse the reported frequencies and percentages as well as compute means and standard deviations. Paired $t$-test was used to compare differences between the means, with statistical significance set at $\mathrm{p}<0.05 .95 \%$ confidence intervals $(\mathrm{Cls})$ of the mean differences were also presented.
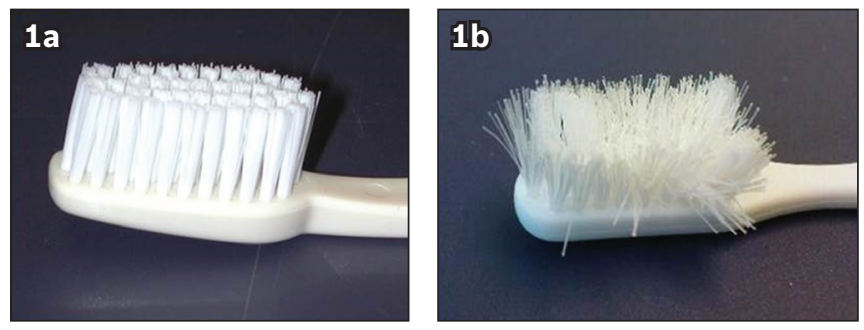

Fig. 1 Photographs of (a) new and (b) used toothbrushes that were used to illustrate normal/healthy and damaged cochlear hair cells, respectively.

Table I. Patients' THI categories at the initial counselling and follow-up sessions $(n=203)$.

\begin{tabular}{|lll|}
\hline \multirow{2}{*}{ THI category } & \multicolumn{2}{c|}{ No. (\%) } \\
\cline { 2 - 3 } & Initial counselling & Follow-up session \\
\hline No handicap & $53(26.1)$ & $146(71.9)$ \\
\hline Mild handicap & $67(33.0)$ & $34(16.7)$ \\
\hline Moderate handicap & $32(15.8)$ & $12(5.9)$ \\
\hline Severe handicap & $51(25.1)$ & $11(5.4)$ \\
\hline
\end{tabular}

THI: Tinnitus Handicap Inventory

\section{RESULTS}

A total of 338 patients were seen at the TCC for the initial session and all were given follow-up appointments. However, only 203 (60.1\%; 83 female, 120 male) patients with tinnitus attended follow-up. Patients who did not attend follow-up were excluded from analysis.

The mean age of the patients was $49.4 \pm 13.7$ (range 18-80) years. The mean time from initial consultation to follow-up was 2.8 (range 1-8) months. The follow-up session was usually scheduled three months after the initial session and there was only one follow-up session after the initial session. It is usual for our ENT doctors to also give patients a review appointment for ENT consultation. Some patients preferred to schedule their ENT consultation and tinnitus counselling appointment on the same day and thereafter changed the appointment for tinnitus counselling. Some patients also requested for a change of appointment for various personal reasons.

$36(17.7 \%)$ patients had normal hearing thresholds within $25 \mathrm{dBHL}$ bilaterally from $250 \mathrm{~Hz}$ to $8,000 \mathrm{~Hz}$, and 90 (44.3\%) patients had bilateral hearing loss of more than $30 \mathrm{dBHL}$ across 4,000-8,000 Hz. 19 (9.4\%) patients presented with unilateral hearing loss and $58(28.6 \%)$ patients showed symmetrical bilateral hearing loss of more than $30 \mathrm{dBHL}$ between $250 \mathrm{~Hz}$ and 8,000 Hz.

At the initial counselling session, 53 (26.1\%) patients had no tinnitus handicap. During the follow-up, an additional 93 (45.8\%) patients were categorised as having no handicap. $40.9 \%$ of patients were in the moderate to severe $\mathrm{THI}$ handicap categories at the initial session. During follow-up, $11.3 \%$ of patients remained in these two categories (Table I). There was a statistically significant difference of 21.9 (95\% Cl 19.1-24.7) between the initial THI score ( $36.1 \pm 23.7$ points) and the THI score at followup (14.2 \pm 18.2 points).

A majority ( $n=198,97.5 \%$ ) of the patients found the initial counselling session helpful. 186 (91.6\%) patients said tinnitus had 


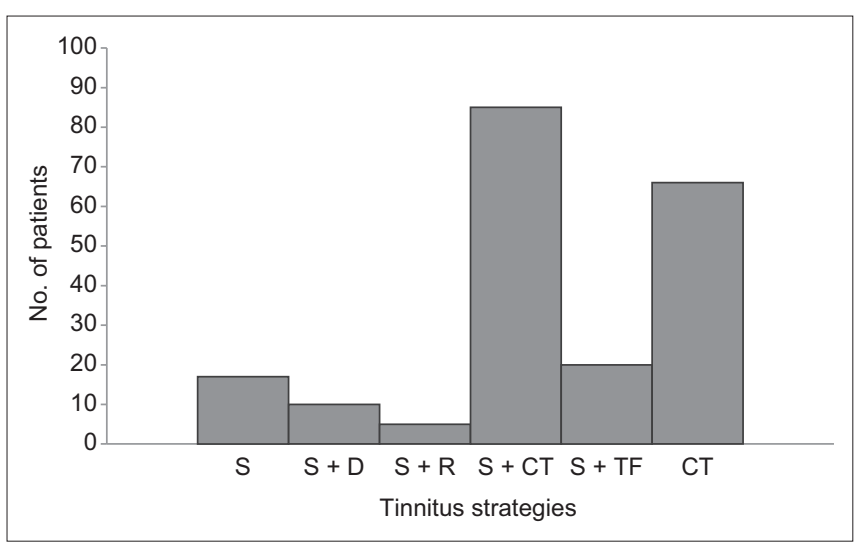

Fig. 2 Chart shows self-help strategies, such as sound and non-sound strategies, chosen by patients for tinnitus management $(n=203)$. $\mathrm{CT}$ : change of thinking; D: distraction; R: relaxation; S: sound enrichment; TF: detection of trigger factors

become more acceptable after counselling. However, 92 (45.3\%) patients reported that they were still seeking a cure for their tinnitus. Patient ratings for counselling components are presented in Table II. The two most helpful counselling components were explanation of self-help strategies and illustration of healthy and damaged hair cells.

Out of 203 patients, 83 (40.9\%) adopted a single strategy for their tinnitus management; 66 (32.5\%) patients said they were able to change their perception of tinnitus after the initial session and $17(8.4 \%)$ patients relied solely on the sound strategy for their tinnitus management (Fig. 2). More than $60 \%$ of patients reported using a combination of sound and non-sound strategies. The most popular choice for self-help was using a sound strategy plus change of thinking, at $41.9 \%(n=85)$.

\section{DISCUSSION}

This study was conducted in the largest TCC in Singapore, with the tinnitus counselling conducted by a trained tinnitus counsellor. The protocol used in the TCC was similar to that of many tinnitus clinics, and included patient education on the condition and introduction to a range of self-help coping strategies. ${ }^{(14-18)}$ The main finding was that a majority of patients $(96.0 \%)$ who returned for a follow-up visit at the TCC found counselling useful. The patients had a better understanding of their condition and were able to take a more active role in managing it, utilising both the sound and non-sound strategies learnt at the TCC.

Many patients with tinnitus who seek help have limited knowledge of how we hear, interpret and react to sounds. Therefore, background information about the auditory pathway and hearing mechanisms must first be provided. Many patients felt that the visual impact of pictures and simple explanation using tools, such as toothbrushes, provided valuable insight.

Patients frequently obtain health information from the Internet. However, the numerous self-help and tinnitus therapy options that are available online can be confusing. Irrespective of the kind of treatment and management given for tinnitus, there is a general consensus that counselling is an essential additional approach to reduce patients' negative reactions to tinnitus and temper their expectations of a cure. The usefulness of counselling
Table II. Patient ratings for how helpful counselling components were $(n=203)$.

\begin{tabular}{|c|c|}
\hline Counselling component & Score* \\
\hline $\begin{array}{l}\text { Explanation of sound and non-sound self-help } \\
\text { strategies }\end{array}$ & $4.40 \pm 0.68$ \\
\hline $\begin{array}{l}\text { Pictures of healthy/damaged hair cells with } \\
\text { toothbrush analogy }\end{array}$ & $4.30 \pm 0.74$ \\
\hline Interpretation of audiogram & $4.23 \pm 0.72$ \\
\hline Written recommendations & $4.21 \pm 0.73$ \\
\hline $\begin{array}{l}\text { Introduction on ear and hearing, tinnitus causes } \\
\text { and theories }\end{array}$ & $4.17 \pm 0.86$ \\
\hline THI and tinnitus history taking & $4.04 \pm 0.73$ \\
\hline
\end{tabular}

*Scores were rated on a 5-point scale (ranging from 1: least helpful to 5: most helpful) and are expressed as mean \pm standard deviation; THI:Tinnitus Handicap Inventory

as a management tool for patients with tinnitus has been well documented. ${ }^{(10,14-17)}$

Patients who are troubled by tinnitus want to learn how to get rid of it. In this study, most patients appreciated information on tinnitus strategies. This was previously noted by Tyler, who believed that in all counselling interventions, there should be a focus on providing strategies. ${ }^{(14)}$ So far, to our knowledge, no one has found a perfect strategy that would be effective for all patients with tinnitus. Therefore, information on the various treatment options available for tinnitus was provided at the TCC as relief rather than cure. Patients were also educated on the sound and non-sound strategies that could be adopted, and how these could be applied in their self-help tinnitus management. Our results suggested that providing information on self-help strategies with explanation is an important component that may provide a significant benefit to patients vis-à-vis their management of tinnitus.

In a previous study for the development of a progressive audiological tinnitus management model for elderly patients with tinnitus, patients and clinicians gave feedback that basic information on ear and tinnitus was good enough in primary care, as many patients only want to be given reassurance. ${ }^{(16)}$ However, patients attending a tertiary care clinic require more than basic information. A recent survey on tinnitus treatments offered at a specialist audiology department found that a majority of patients rated counselling and education as more effective than sound devices. ${ }^{(19)}$

Most patients felt that the least useful component of the initial session was completing the THI questionnaire and tinnitus history taking. History taking of the patient's tinnitus is an essential component of any counselling protocol, as: (a) the tinnitus service provider needs to understand the patient's condition in order to customise recommendations; and (b) the THI is needed to evaluate the impact of tinnitus on quality of life, and monitor the changes before and after counselling sessions. Furthermore, history taking and discussion of the THI also acts as an icebreaker that helps to build rapport with the patients.

In our study, most patients who attended follow-up preferred a combination of sound and non-sound strategies for their tinnitus management. The provision of a clear therapy plan with several 
management options allows patients to feel that they can better control their tinnitus. ${ }^{(20)}$ Sound enrichment from the environment and sound devices, such as listening to the radio, provide a practical and natural option for paying less attention and removing some annoyance associated with tinnitus. This progressively promotes their natural habituation to tinnitus. With this informed knowledge, many of our patients were able to make appropriate choices to suit their lifestyles.

Among 83 patients who chose a single self-help strategy after initial counselling, 66 (32.5\%) patients reported adopting change of thinking with respect to tinnitus. We found that patients seeking help at the hospital often fear that tinnitus may be a symptom of something more severe. They tend to worry that it may get worse, wonder if there is a cure or if tinnitus will ever go away. Therefore, counselling on the change of thinking strategy provided our patients reassurance and hope, and also gave them new and positive expectations regarding the condition.

Many patients felt relieved to share their experience with someone who was knowledgeable about tinnitus and who could take the mystery out of tinnitus. ${ }^{(20)}$ Even though more than half of the follow-up patients remained hopeful for a cure for tinnitus at follow-up, a majority of our patients did express tinnitus acceptance after counselling. Some written supportive statements from our patients are as follows:

- $\quad$ "Counselling provides an in-depth understanding of tinnitus and ways it can be managed, also knowing that tinnitus has no harmful effect on an individual. Great!"

- Patient 1, a 48-year-old man with tinnitus for 19 months

- "Counselling helps me to get over the problem, giving me more information and insight to the problem. These will probably help me in the long run, for years to come."

- Patient 2, a 22-year-old man with tinnitus for one year

- $\quad$ "The session is informative. It set my mind at ease. Knowing it is part of me now, I have to learn to adapt and accept the noise."

- Patient 3, a 39-year-old woman with tinnitus for three weeks

However, one limitation of our study was that these responses were obtained only from patients who attended follow-up. A random check by telephone interview of 20 patients who did not attend follow-up elicited the following reasons:

- "It is a paid service and quite costly."

- "I cannot be bothered with follow-up, since investigations, such as MR (magnetic resonance) imaging and other investigations were normal."

- "I already accept tinnitus as part of my life, and do not think the follow-up session will make any difference to my tinnitus."

- "There is no cure for tinnitus, so nothing new can be expected at the follow-up."

- "I want to try TCM (traditional Chinese medicine) and acupuncture."

A Korean study reported findings of a telephone survey of patients who did not go for follow-up after an initial counselling session on tinnitus retraining therapy. ${ }^{(21)}$ In their study, most patients were "able to obtain sufficient relief that they no longer felt the need for additional treatment". Over $60 \%$ of patients who attended follow-up were not interested in any treatment and did not plan to have further tinnitus treatment. Some patients in the earlier study felt that the cost and inconvenience of hospital visits was not worth it, as they were already comfortable with their condition. The authors concluded that perhaps a single counselling session on the concept of tinnitus retraining therapy may be sufficient for such patients. ${ }^{(21)}$

Another limitation of our study was that the effectiveness of any of the components presented was not assessed. We hope to address this in future research. Additionally, as ours was a retrospective review of routinely collected operational data and not a prospectively designed study, there were bound to be design limitations. The absence of blinding and interviewer bias were other likely sources of bias.

In conclusion, a majority of patients who returned for followup at the TCC found counselling useful. Of the counselling protocol components, patients most frequently adopted both sound and non-sound strategies to manage tinnitus. Counselling helped promote better understanding of the condition and offered patients the opportunity to take an active role in managing it. Future research may address the effectiveness of each component presented.

\section{ACKNOWLEDGEMENTS}

The authors would like to thank Dr Hsu Pon Poh, Dr Yuen Heng Wai, Dr Steven Lee, Ms Savitha Kamath and Mrs Joanna Wong of the Department of Otolaryngology, CGH, and Dr William Vehoeven, visiting consultant in orthopaedic surgery, $\mathrm{CGH}$, for their support and encouragement.

\section{REFERENCES}

1. Newman CW, Sandridge SA, Bea SM, et al. Tinnitus: patients do not have to 'just live with it'. Cleve Clin J Med 2011; 78:312-9.

2. Jastreboff PJ. 25 years of tinnitus retraining therapy. HNO 2015; 63:307-11.

3. Cima RF, Andersson G, Schmidt CJ, Henry JA. Cognitive-behavioral treatments for tinnitus: a review of the literature. J Am Acad Audiol 2014; 25:29-61.

4. Doi MY, Tano SS, Schultz AR, Borges R, Marchiori LL. Effectiveness of acupuncture therapy as treatment for tinnitus: a randomized controlled trial. Braz J Otorhinolaryngol 2016; 82:458-65

5. Meeus O, De Ridder D, Van de Heyning P. Administration of the combination clonazepam-Deanxit as treatment for tinnitus. Otol Neurotol 2011; 32:701-9.

6. Davis PB, Paki B, Hanley PJ. Neuromonics tinnitus treatment: third clinical trial. Ear Hear 2007; 28:242-59.

7. Seo YJ, Kim HJ, Moon IS, Choi JY. Changes in tinnitus after middle ear implant surgery: comparisons with the cochlear implant. Ear Hear 2015; 36:705-9.

8. Searchfield GD, Kaur M, Martin WH. Hearing aids as an adjunct to counseling: tinnitus patients who choose amplification do better than those that don't. Int J Audiol 2010; 49:574-9.

9. Soleimani R, Jalali MM, Hasandokht $T$. Therapeutic impact of repetitive transcranial magnetic stimulation (rTMS) on tinnitus: a systematic review and meta-analysis. Eur Arch Otorhinolaryngol 2016; 273:1663-75.

10. Langguth B. Treatment of tinnitus. Curr Opin Otolaryngol Head Neck Surg 2015; 23:361-8.

11. Lim JJ, Lu PK, Koh DS, Eng SP. Impact of tinnitus as measured by the Tinnitus Handicap Inventory among tinnitus sufferers in Singapore. Singapore Med J 2010; 51:551-7.

12. Lee SY, Kim JH, Hong SH, Lee DS. Roles of cognitive characteristics in tinnitus patients. J Korean Med Sci 2004; 19:864-9.

13. Newman CW, Jacobson GP, Spitzer JB. Development of the Tinnitus Handicap Inventory. Arch Otolaryngol Head Neck Surg 1996; 122:143-8.

14. Tyler R. Tinnitus Treatment. In: Tyler RS, ed. Neurophysiological Models, Psychological Models, and Treatments for Tinnitus. New York: Thieme, 2006: 1-22.

15. Sweetow RW. An Integrated Approach to Tinnitus Management. In: 
AudiologyOnline. Available at: http://www.audiologyonline.com/articles/ integrated-approach-to-tinnitus-management-11598. Accessed February 4, 2013.

16. Myers PJ, Griest S, Kaelin C, et al. Development of a progressive audiologic tinnitus management program for Veterans with tinnitus. J Rehabil Res Dev 2014; 51:609-22.

17. Tyler RS, Bergan CJ. Tinnitus retraining therapy: a modified approach. Hear J $2001 ; 54: 36$.

18. Gudex C, Skellgaard PH, West T, Sørensen J. Effectiveness of a tinnitus management programme: a 2-year follow-up study. BMC Ear Nose Throat Disord
2009; 9:6.

19. Aazh H, Moore BC, Lammaing K, Cropley M. Tinnitus and hyperacusis therapy in a UK National Health Service audiology department: patients' evaluations of the effectiveness of treatments. Int J Audiol 2016; 55:514-22.

20. Tyler R, Haskell G, Preece J, Bergan C. Nurturing patient expectations to enhance the treatment of tinnitus. Semin Hear 2001; 22:15-22.

21. Han JJ, Lee JH, Oh SH, Chang SO, Suh MW. Assessing the effects of tinnitus retraining therapy in patients lost to follow-up: a telephone survey. Otol Neurotol $2015 ; 36: 581-7$. 